\title{
The effect of citrus flavonoid extract supplementation on anaerobic capacity in moderately trained athletes: a randomized controlled trial
}

\author{
Lieke E. van lersel ${ }^{1+}$, Yala R. Stevens ${ }^{1,2^{*}+}$ (D) Jose M. Conchillo ${ }^{2}$ and Freddy J. Troost ${ }^{2,3}$
}

\begin{abstract}
Background: Nutritional supplementation is commonly used by athletes to improve their exercise performance. Previous studies demonstrated that citrus flavonoid extract (CFE) supplementation may be an effective strategy to improve exercise performance in male athletes. Yet, no conclusive research has been performed to investigate the effect of chronic CFE supplementation on high-intensity exercise performance under anaerobic conditions. Therefore, the aim of the study was to assess whether CFE supplementation in daily dosages of 400 and $500 \mathrm{mg}$ for a period of 4 and 8 weeks improves anaerobic exercise capacity.

Methods: A randomized, double-blind, placebo controlled, parallel clinical study was conducted in 92 moderately trained healthy men and women. Subjects were randomized to receive $400 \mathrm{mg}$ of CFE $(n=30), 500 \mathrm{mg}$ of CFE ( $n=$ 31) or placebo $(n=31)$ daily, for 8 consecutive weeks. The Wingate anaerobic test was used to assess anaerobic exercise capacity and power output at baseline, after 4 weeks and after 8 weeks.

Results: After 4 weeks supplementation, average power output significantly increased in the $400 \mathrm{mg}$ group (Estimated difference $[\mathrm{ED}]=38.2 \mathrm{~W}[18.0,58.3]$; $p<0.001$; effect size $[\mathrm{ES}]=0.27$ ) and in the $500 \mathrm{mg}$ group (ED $=21.2$ $W[0.91,41.4] ; p=0.041 ; E S=0.15)$ compared to placebo. The $5 \mathrm{~s}$ peak power output was also increased in the 400 mg group $(E D=53.6[9.96,97.2] ; p=0.017 ; E S=0.25)$ after 4 weeks compared to placebo. After 8 weeks of supplementation, average power output was significantly improved in the group receiving $400 \mathrm{mg}$ of CFE (ED = $31.6[8.33,54.8] ; p=0.008 ; E S=0.22$ ) compared to placebo.

Conclusion: These results demonstrate that CFE supplementation improved anaerobic capacity and peak power during high intensity exercise in moderately trained individuals. Further research is needed to identify the underlying mechanisms that are affected by CFE supplementation.
\end{abstract}

Trial registration: ClinicalTrials.gov (NCT03044444). Registered 7 February 2017

Keywords: Antioxidant, Wingate anaerobic test, Anaerobic capacity, Hesperidin

\footnotetext{
* Correspondence: yala.stevens@maastrichtuniversity.nl

${ }^{+}$Lieke E. van lersel and Yala R. Stevens contributed equally to this work.

'BioActor BV, Gaetano Martinolaan 85, 6229 GS Maastricht, The Netherlands

2Division Gastroenterology-Hepatology, Department of Internal Medicine,

School of Nutrition and Translational Research in Metabolism (NUTRIM),

Maastricht University, P.O. Box 616, 6200 MD Maastricht, The Netherlands

Full list of author information is available at the end of the article
}

C C The Author(s). 2021 Open Access This article is licensed under a Creative Commons Attribution 4.0 International License, which permits use, sharing, adaptation, distribution and reproduction in any medium or format, as long as you give appropriate credit to the original author(s) and the source, provide a link to the Creative Commons licence, and indicate if changes were made. The images or other third party material in this article are included in the article's Creative Commons licence, unless indicated otherwise in a credit line to the material. If material is not included in the article's Creative Commons licence and your intended use is not permitted by statutory regulation or exceeds the permitted use, you will need to obtain permission directly from the copyright holder. To view a copy of this licence, visit http://creativecommons.org/licenses/by/4.0/ The Creative Commons Public Domain Dedication waiver (http://creativecommons.org/publicdomain/zero/1.0/) applies to the data made available in this article, unless otherwise stated in a credit line to the data. 


\section{Introduction}

Nutritional supplementation is commonly used by athletes to improve their exercise performance [1]. Supplementation with vitamins, antioxidants, creatine, proteins and carbohydrates aiming at enhancing exercise performance and muscle recovery has become of great interest in recent years, as these supplements may have beneficial effects on metabolic activity, strength and overall exercise performance [2-4]. During exercise, skeletal muscle mitochondria are of pivotal importance for the production of energy in the form of adenosine triphosphate (ATP), which is produced trough mitochondrial respiration. However, during the first $30 \mathrm{~s}$ of very high intensity-exercise, the phosphagen system is involved, producing immediate but limited energy under anaerobic conditions $[5,6]$.

It is considered that moderate exercise induces production of low concentrations of reactive oxygen species (ROS) in mitochondria, which subsequently act as a signal to stimulate the synthesis of enzymes that are involved in the adaptive response of the skeletal muscle, such as nitric oxide (NO) synthase and superoxide dismutase (SOD) [7-9]. However, the beneficial effects of low ROS levels will be lost when physical activity becomes strenuous [8]. During high intensity exercise, muscles rely at least in part on anaerobic metabolism. Anaerobic energy expenditure increases the production of fatigue associated products, mainly lactate and high levels of ROS, which contributes to impaired muscle functioning and oxidative damage as the body's antioxidative scavenging capacity may be exceeded $[2,5$, 6]. Subsequently, the oxidant/antioxidant balance becomes skewed in favor of free radical production, resulting in oxidative stress, structural cell damage and potentially also inflammation [8].

To prevent the negative consequences of excessive free radical accumulation during high intensity exercise, daily polyphenol supplementation can be an effective strategy to enhance muscle strength and improve muscle recovery. Polyphenols are compounds, which are naturally present in plants. They can be divided into different classes, based on their chemical structure, of which flavonoids are the largest group. Recent studies have shown anti-inflammatory and antioxidant effects of polyphenols which are present in fruits, such as blueberries, cherries, pomegranate and citrus fruit $[10,11]$. In addition, a clinical study with polyphenol-rich extract supplementation showed an increased maximal power output, average power and total power output during high intensity exercise, without inducing more fatigue [2]. Furthermore, a translational study demonstrated that citrus flavonoid treatment improved vascular function by reducing circulating inflammatory biomarkers and stimulation of NO production [12]. It is suggested that increased NO production is associated with endothelium-dependent vasodilation in the arterial wall, causing a reduction in blood pressure. In relation with exercise physiology, blood flow to active muscles will be improved [11]. Hereby, fatigue-related metabolites are removed quickly and nutrient and oxygen delivery to active muscles is enhanced, improving tolerance to physical exercise and muscle recovery mechanisms [13].

A previous study with flavonoids from citrus fruit showed that repeated intake of $500 \mathrm{mg}$ of a specific citrus flavonoid extract (CFE) improved aerobic exercise performance during a ten-minute bicycle trial on an ergometer. An increase in absolute power output and decrease in oxygen consumption/power ratio in trained male athletes was observed after 4 weeks of CFE treatment [10]. Furthermore, acute intake of a single dose of $500 \mathrm{mg}$ CFE resulted in an increase in average power, maximum speed and total energy during the best sprint in an anaerobic cycling performance test, the Wingate Test [14]. However, it is unknown if chronic CFE supplementation also affects anaerobic exercise performance and whether such effects may also be achieved by lower daily supplementation dosages.

Therefore, the aim of the present clinical study was to investigate whether supplementation with CFE at dosages of $400 \mathrm{mg}$ and $500 \mathrm{mg}$ for a duration of 4 and 8 consecutive weeks improves anaerobic physical performance in moderately trained athletes during a Wingate Anaerobic Test (WAnT). It was hypothesized that CFE supplementation improves anaerobic exercise capacity in moderately trained individuals.

\section{Methods}

The study was approved by the Medical Ethics Committee of Wageningen University, Wageningen, The Netherlands, and was conducted in full accordance with the principles of the Declaration of Helsinki of 1975 as amended in 2013 (Fortaleza, Brazil) and with the Dutch Regulations on Medical Research involving Human Subjects (1998). All participants gave written informed consent before participation. The study was performed at Topsport Limburg High Performance Center, Sittard, The Netherlands between April 2017 and April 2018 and has been registered at clinicaltrials.gov (NCT03044444).

\section{Subjects}

Healthy, non-smoking, moderately trained volunteers, both male and female, aged 18-35 years and Body Mass Index (BMI) between 18 and $30 \mathrm{~kg} / \mathrm{m}^{2}$ were recruited through advertisements in local media and through partnership with Topsport Limburg High Performance Center, Sittard, The Netherlands. Participants were amateur athletes in resistance or interval sports, engaging in 
moderate physical activity for a minimum of $4 \mathrm{~h}$ a week. Exclusion criteria were as follows: the use of medication and ergogenic supplements (e.g. creatine or anabolic steroids) that may interfere with study outcomes; intake of antioxidant/vitamin supplements; cardiovascular complications; allergy to study product or placebo; intake of dietary products containing citrus flavonoids or metabolites; and abuse of alcohol (>20 alcoholic units/wk) or recreational drugs.

\section{Design and protocol}

The study was designed as a randomized, double-blind, placebo-controlled parallel study to test the effects of a daily dosage of $400 \mathrm{mg}$ CFE supplementation or $500 \mathrm{mg}$ CFE supplementation versus placebo over a period of 4 and 8 consecutive weeks (Fig. 1). Previous human intervention studies focusing on exercise performance or other relevant outcomes such as endothelial function have been performed using a citrus flavonoid (extract) supplementation dosage of $500 \mathrm{mg}[10,12,14,15]$. However, so far, information about the effectiveness at lower dosages is limited. The present study aimed to test whether the same putative performance outcomes over the 8-weeks supplementation period can be achieved with a lower dosage of CFE supplementation, as this would benefit future applications. To the best of our knowledge, this is the first human study investigating the effects of long-term CFE supplementation on anaerobic physical performance at a daily dose of $500 \mathrm{mg}$, as well a 20\% lower daily dose of $400 \mathrm{mg}$.

Participants were randomly assigned to one of the following three intervention groups: CFE $400 \mathrm{mg}$, CFE 500 $\mathrm{mg}$ or placebo. A randomization list was generated by an independent person using a randomization program (http://randomizer.org). The study consisted of a familiarization test, a baseline test (visit 1), a test day after 4 weeks of intervention (visit 2) and a test day after 8 weeks of intervention (visit 3). During the intervention period, participants ingested CFE $400 \mathrm{mg}$, CFE $500 \mathrm{mg}$ or placebo supplements q.d. (once per day). Subjects were instructed to abstain from high intensity exercise and consumption of caffeine and alcohol $24 \mathrm{~h}$ prior to each test day. During the entire study period, participants were instructed to refrain from consuming foods containing high levels of citrus flavonoids (i.e. lemons, oranges and grapefruit) and to maintain their habitual dietary intake and weekly training schedule. At the start of each test visit, subjects handed in a 3-day dietary record.

The familiarization test was performed in order to get familiar with the testing procedures and exercise protocol. At the familiarization test, subjects performed the WAnT, preceded by a standardized warmup protocol. The test protocol was performed identically on each test day.

\section{Wingate anaerobic test}

The WAnT was performed on a Wattbike Performance Monitor (WPM) (Wattbike Ltd., Nottingham, UK). Before warm-up, the subjects were provided with a Garmin HRM1G-Heart Rate Monitor. During the familiarization test the optimal settings for saddle height, saddle position and steer height and position were determined and recorded. The resistance level was calculated based on gender and bodyweight by the WPM [16]. The resistance was set at $0.095 \mathrm{~kg} / \mathrm{kg}$ bodyweight for men and $0.086 \mathrm{~kg} / \mathrm{kg}$ bodyweight for women [17]. The warm-up consisted of $14 \mathrm{~min}$ of cycling at $\pm 100 \mathrm{~W}$. After 10, 11

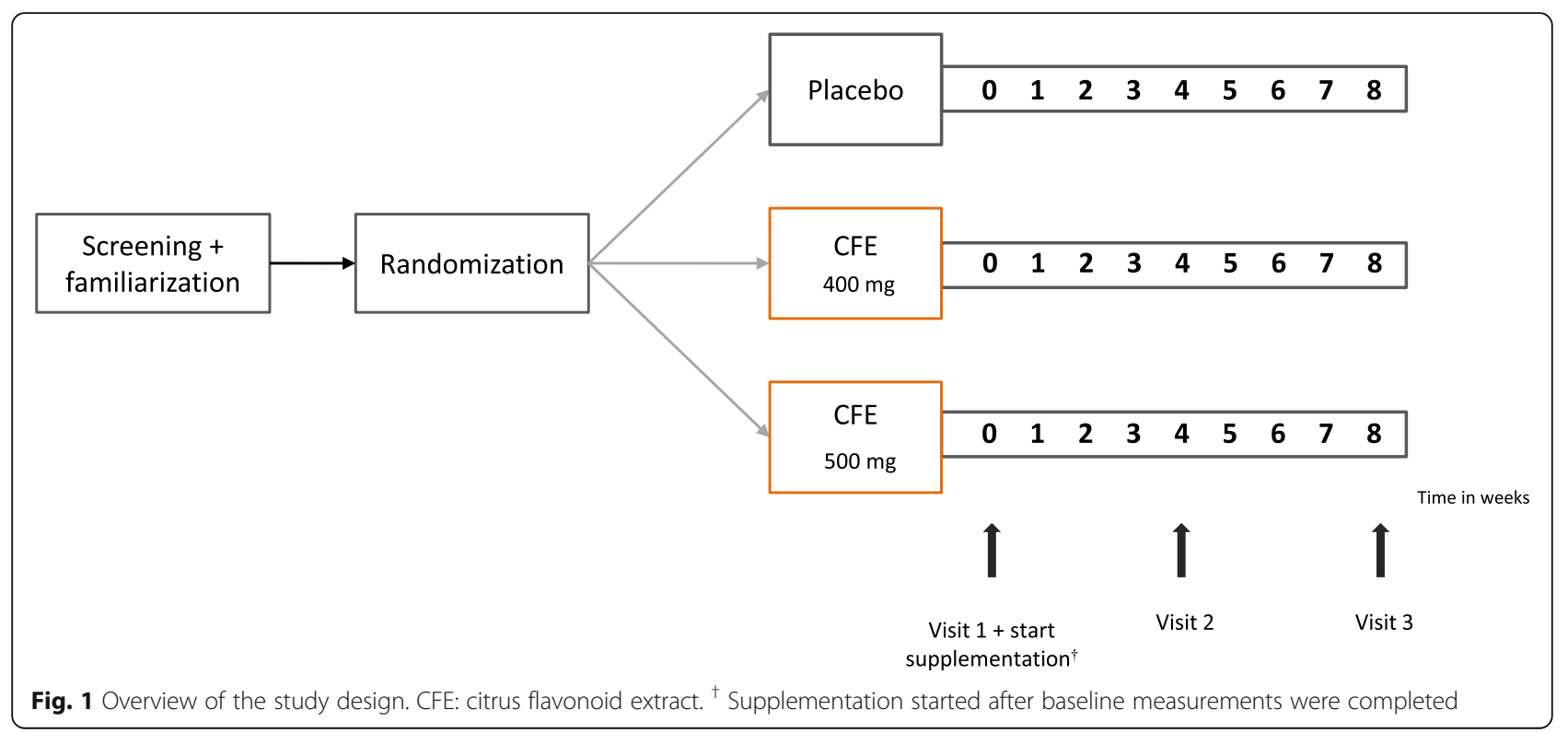


and $12 \mathrm{~min}$, the subjects were requested to sprint all-out for $3 \mathrm{~s}$. Subsequently, subjects rested for exactly $1 \mathrm{~min}$ and after a $5 \mathrm{~s}$ countdown, the subjects started to sprint for 30 consecutive seconds, conform to the standard WAnT protocol [18]. They were instructed to generate the maximal total power output (W) possible over the time course of $30 \mathrm{~s}$. After $30 \mathrm{~s}$, the resistance was set back and the subject cycled on slow pace for $3 \mathrm{~min}$ after which the test was finished. The WPM was interfaced with Wattbike Expert Ver. 2.60.20 (Wattbike Ltd., Nottingham, UK), which assessed average power (AP) and 5 $\mathrm{s}$ peak power (5sPP) to determine anaerobic capacity and anaerobic peak power. In addition, Time to Peak in seconds was measured and heart rate (HR) in beats per minute (bpm) was monitored continuously over the $30 \mathrm{~s}$ sprint trial, to assess the maximum HR.

\section{Study product}

The test product is a Citrus sinensis extract containing $90 \%$ hesperetin-7-O-rutinoside of which $>75 \%$ is comprised of the $2 \mathrm{~S}$ enantiomer (WATTS'UP ${ }^{\circledR}$, BioActor BV, Maastricht, the Netherlands). Microcrystalline cellulose (Microz, Geleen, the Netherlands) was used as placebo. The study products were formulated into capsules. The placebo and CFE500 contained $250 \mathrm{mg}$ study product per capsule, the CFE400 capsules contained $200 \mathrm{mg}$ study product and $50 \mathrm{mg}$ microcrystalline cellulose. Furthermore, the study products were produced to be identical in flavor and appearance. The subjects were instructed to orally ingest two capsules with $200 \mathrm{~mL}$ water each morning before consuming breakfast. The total amount of hesperidin in the CFE400 and CFE500 supplements contained the equivalent of approximately $0.7-0.9 \mathrm{~L}$ of commercially available sweet orange juice [19].

\section{Dietary intake}

Dietary intake was assessed by 3-day dietary records as described previously [20]. Briefly, participants were asked to record the intake of 2 week days and one weekend day before each test day, based on standard household units.

\section{Statistical analysis}

Statistical analysis was performed using IBM SPSS Statistics for Windows (version 25.0, IBM Corporation, Armonk, NY, USA). The sample size was determined with a significance level of $\alpha=0.05$ and a power of $90 \%$. Based on previous work [10], a sample size of 78 participants was required to reach sufficient statistical power. Data are presented as observed mean \pm standard deviation (SD) for numerical variables and numbers for categorical variables. Differences in outcomes between the intervention groups (i.e. CFE400 and CFE500) and the placebo were assessed by unstructured linear mixed model analyses. Intervention, time and intervention $\mathrm{x}$ time were included as fixed factors. This model accounts for the correlation between repeated measures and missing data. Intention to treat analyses were performed for all outcomes. The estimated mean difference in change scores from baseline between the intervention groups and the placebo [95\% confidence interval] obtained from this model are reported, matching with the intention to treat analysis and taking missing data into account. The effect size (ES) was determined by dividing the estimated mean difference by the square root of the estimated baseline residual variance obtained from the model. Within-group differences were not tested. Level of significance was set on $p<0.05$.

\section{Results}

Study subjects

In total, 93 healthy subjects were enrolled in the study. Seventy-nine subjects completed the exercise test (WAnT) after 4 weeks supplementation as well as after 8 weeks supplementation. One subject, allocated to the CFE400 group, did not start the study due to private circumstances Thirteen subjects did not complete the second test day after 4 weeks of supplementation due to injuries or illness not related to the study. In total, 79 subjects completed the third test day after 8 weeks of supplementation. (Fig. 2). Baseline characteristics of the study population are shown in Table 1 .

\section{Average power output after 4 weeks}

No significant differences in body weight were observed over time in the placebo and the two treatment groups. Therefore, the performance outcomes were not corrected for body weight. The results of the average power output from the participants supplemented with CFE and placebo, before and after 4 weeks intervention are summarized in Table 2 and Fig. 3. When comparing the differences in AP output between the CFE400 group and placebo, a significant effect $(p<0.001 ; \mathrm{ES}=0.27)$ was found after 4 weeks of treatment. The CFE500 supplementation also resulted in a significant difference in AP output versus placebo $(p=0.041 ; \mathrm{ES}=0.15)$.

\section{Peak power output after 4 weeks}

The results of the peak power output from the participants supplemented with CFE and placebo, before and after 4 weeks intervention are summarized in Table 2 and Fig. 3 . When comparing the differences in 5sPP between baseline and after 4 weeks of supplementation, a significant effect between the CFE400 group and placebo was found $(p=0.017$; $\mathrm{ES}=0.25)$, but no significant difference in $5 \mathrm{sPP}$ was found in the intervention group receiving CFE500 versus placebo $(p=0.197$; ES $=0.13)$. 


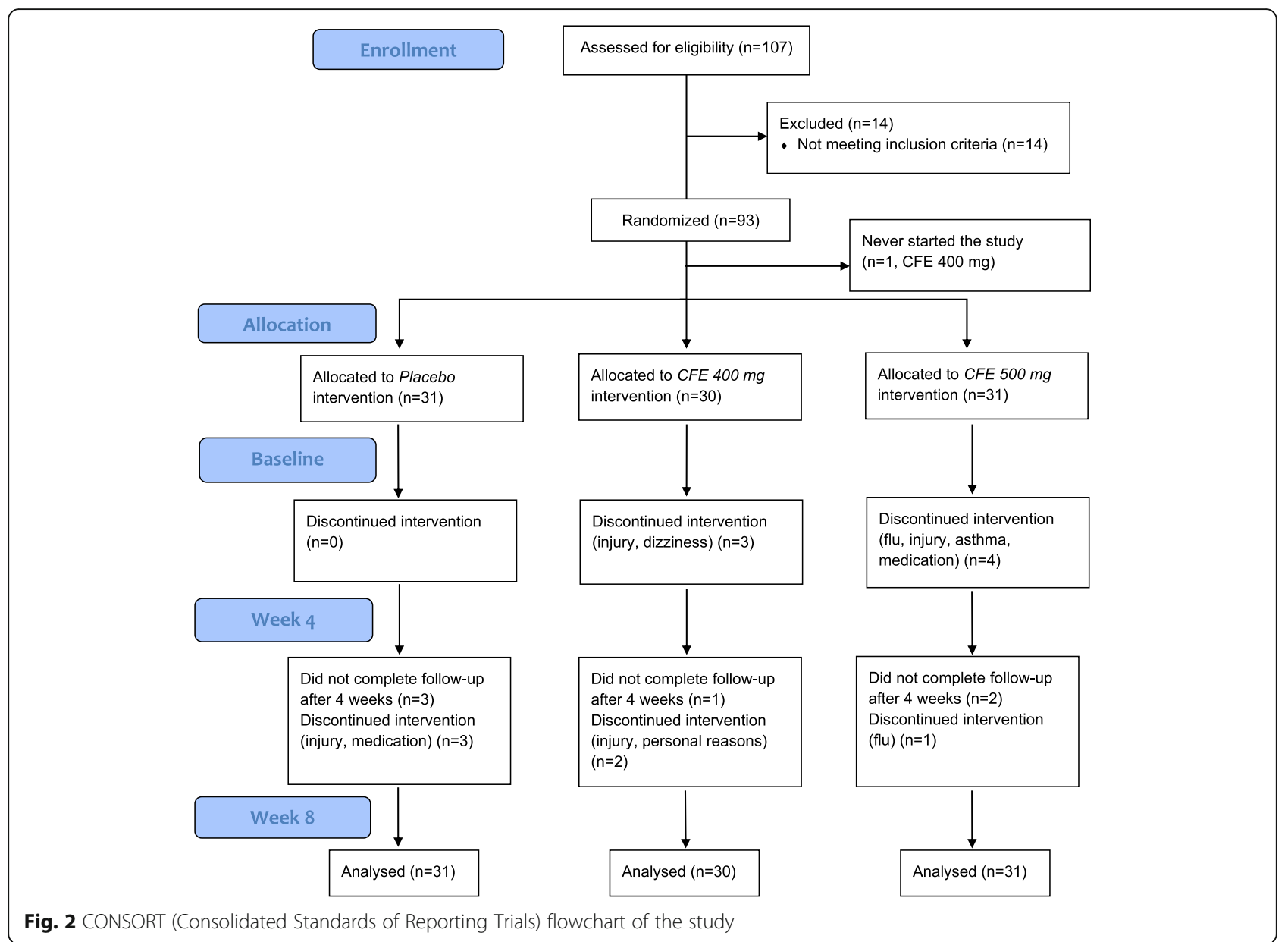

Additionally, a slight decrease in Time to peak was observed after 4 weeks in the CFE400 group compared to the placebo $(p=0.060 ; \mathrm{ES}=-0.50)$. No changes in HR were observed between the groups after 4 weeks of supplementation.

\section{Average power output and peak power output after 8 weeks}

AP output improved significantly in the CFE400 group compared to placebo after 8 weeks of supplementation $(p=0.008 ; \mathrm{ES}=0.22$; Table 3; Fig. 3). Supplementation

Table 1 Baseline participant characteristics

\begin{tabular}{|c|c|c|c|c|c|}
\hline & $\begin{array}{l}\text { Total } \\
(N=92)\end{array}$ & $\begin{array}{l}\text { Placebo } \\
(N=31)\end{array}$ & $\begin{array}{l}\text { CFE400 } \\
(N=30)\end{array}$ & $\begin{array}{l}\text { CFE500 } \\
(N=31)\end{array}$ & $P$-value \\
\hline Male/Female $(N)$ & $52 / 40$ & 20/11 & $15 / 15$ & $17 / 14$ & 0.506 \\
\hline Age (years) & $24 \pm 5$ & $24 \pm 5$ & $24 \pm 5$ & $23 \pm 4$ & 0.361 \\
\hline Weight (kg) & $70.8 \pm 11.0$ & $71.9 \pm 11.5$ & $68.7 \pm 11.7$ & $71.7 \pm 9.8$ & 0.445 \\
\hline Height (m) & $1.77 \pm 0.1$ & $1.77 \pm 0.1$ & $1.76 \pm 0.1$ & $1.79 \pm 0.1$ & 0.275 \\
\hline $\mathrm{BMI}\left(\mathrm{kg} / \mathrm{m}^{2}\right)$ & $22.4 \pm 2.2$ & $23.0 \pm 2.0$ & $22.2 \pm 2.3$ & $22.2 \pm 2.2$ & 0.262 \\
\hline Exercise time (h) & $7.94 \pm 4.15$ & $8.11 \pm 4.6$ & $7.63 \pm 3.6$ & $8.07 \pm 4.4$ & 0.885 \\
\hline Average Power (W) & $543 \pm 143$ & $547 \pm 141$ & $511 \pm 134$ & $571 \pm 149$ & 0.259 \\
\hline 5 s Peak Power (W) & $721 \pm 215$ & $726 \pm 228$ & $675 \pm 200$ & $762 \pm 214$ & 0.286 \\
\hline Time to peak (s) & $1.73 \pm 2.2$ & $1.57 \pm 3.0$ & $2.37 \pm 1.9$ & $1.28 \pm 1.1$ & 0.131 \\
\hline Heart Rate Maximum (BPM) & $173 \pm 11$ & $171 \pm 9$ & $173 \pm 14$ & $173 \pm 10$ & 0.699 \\
\hline
\end{tabular}

Values are presented as mean \pm SD. Age, Weight, Height, BMI, Exercise time, Average Power, $5 \mathrm{~s}$ Peak Power, Time to peak and Heart Rate Maximum were compared between intervention groups with the use of a one-way analysis of variance (ANOVA). Gender was compared between intervention groups with the use of a Pearson's chi-square test. BMI for all participants was between 19.40 and $29.10 \mathrm{~kg} / \mathrm{m}^{2}$. CFE Citrus Flavonoid Extract. BMI Body Mass Index 


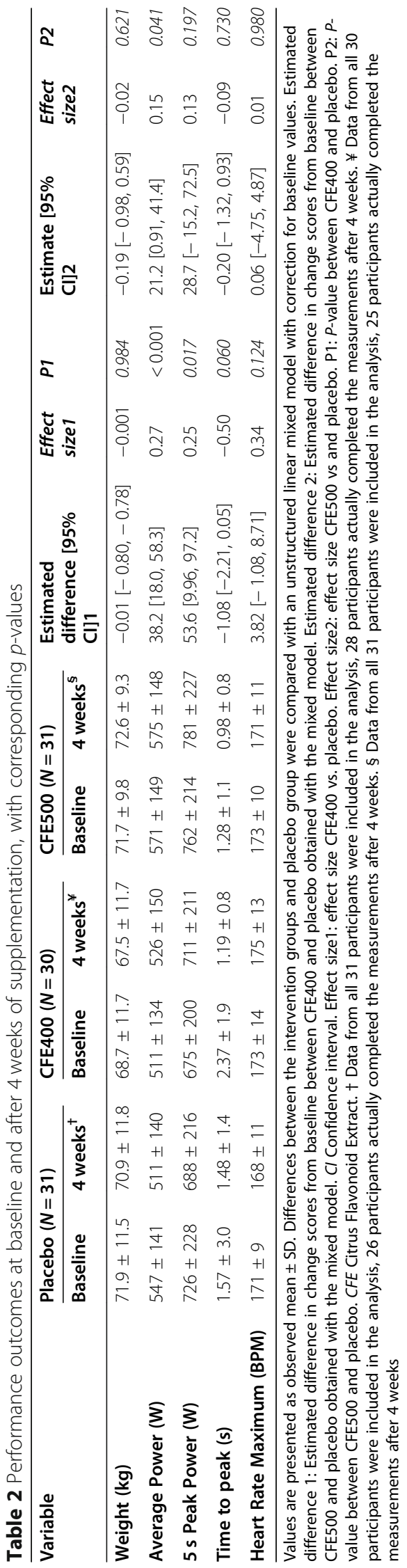



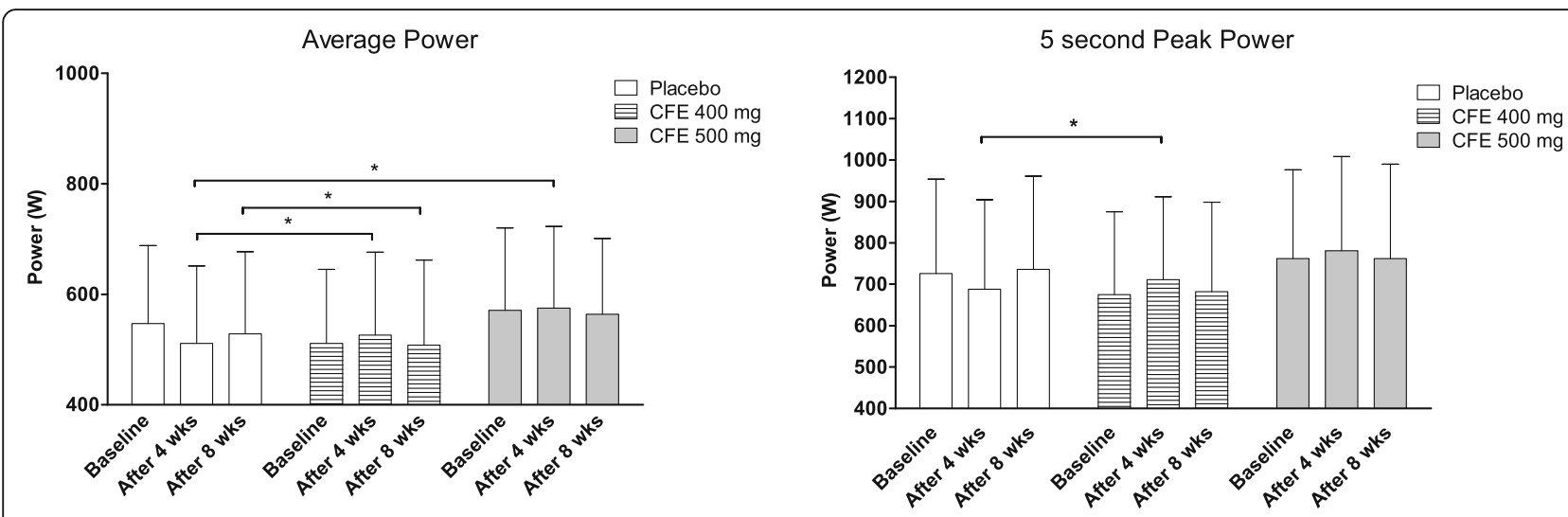

Fig. 3 Performance outcomes at baseline, after 4 weeks and after 8 weeks of supplementation. Values are presented as observed mean \pm SD. Differences between the intervention groups and placebo group were compared with an unstructured linear mixed model with correction for baseline values. ${ }^{*} p<0.05$. CFE: Citrus Flavonoid Extract

with CFE500 for 8 weeks did not result in significant differences in AP output versus placebo $(p=0.471$; ES = $0.06)$. No significant changes in $5 \mathrm{sPP}$ were found in the CFE400 group nor the CFE500 group versus placebo after 8 weeks of intervention (Table 3; Fig. 3).

\section{Dietary intake}

The intake of energy and macronutrients of the participants was assessed during the intervention period (Table s1). No significant differences in dietary intake (not in total energy intake, nor in fat-, protein-, and carbohydrate intake) between the intervention groups were observed throughout the study period.

\section{Discussion}

This study determined the effects of 8-weeks CFE supplementation in different dosages on exercise performance under anaerobic conditions in moderately trained individuals. Both CFE dosages (400 and $500 \mathrm{mg}$ q.d., respectively) significantly increased anaerobic exercise performance as assessed by average power output during the Wingate test after 4 weeks, compared to placebo. In the CFE400 group, but not in the CFE500 group, this was accompanied by a significant increase in peak power output, as indicated by the 5sPP. After 8 weeks of supplementation, the anaerobic exercise performance was still increased in the CFE400 group compared to placebo. No differences were observed after 8 weeks of CFE500 supplementation.

The effect of polyphenols or antioxidants on endurance exercise performance has been investigated in previous studies, demonstrating that polyphenol supplementation can improve performance outcomes in study populations ranging from healthy but untrained individuals to trained athletes [10, 21-24]. However, research into the effects of polyphenol supplementation on anaerobic capacity is less extensive. In the present study, we showed that daily supplementation with CFE400 increased anaerobic capacity in moderately trained individuals. After 4 weeks of CFE supplementation, AP was also significantly improved in the CFE500 group and the 5sPP output increased in the CFE400 group, both compared to placebo. This increase did not result in a concomitant increase in maximum heart rate during the exercise.

The outcomes of 4 weeks CFE treatment are in line with those from previous studies. In male amateur cyclists, a single dose of CFE administered $5 \mathrm{~h}$ before the exercise session increased average power by $2.3 \%$, maximum speed by $3.2 \%$ and total energy by $2.6 \%$ when data of the best of 4 sprints was considered. No significant improvements of this acute dose were observed for the average results [14]. In a study investigating supplementation of a polyphenol-rich extract, peak power was shown to be increased by $3.7 \%$ and average power by $5 \%$, without a concomitant increase in maximum HR [2]. That study demonstrated that polyphenol supplementation did not affect heart workload despite the increased power outputs, probably due to a decreased blood pulse pressure, which is considered to be a biomarker for heart workload [2, 25]. Despite the fact that blood pulse pressure was not measured in the current study, the same results in HR were observed. It might be possible that CFE supplementation decreased intravenous resistance as some studies showed that polyphenol supplementation is related to an increased endothelial NO production $[12,13]$. In the present study, a higher dosage of CFE did not result in better performance output, as the CFE 400 overall showed a stronger improvement in exercise performance outcomes than the CFE500 compared to placebo both after 4 and after 8 weeks of supplementation. This finding was surprising, as we were expecting no difference in performance. As such, it is tempting to speculate on the possible 


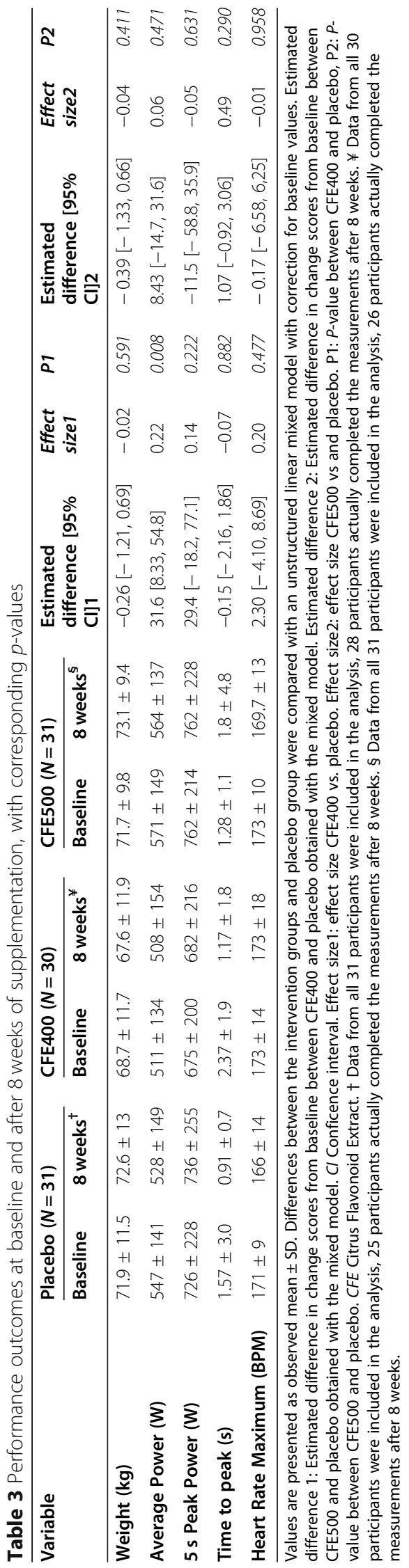


explanation for these findings. One potential explanation might be that the higher dosage may have reduced the ROS concentrations within the skeletal muscle to a larger extent, resulting in concentrations that were less optimal for muscle contractility [26].

We included healthy, moderately trained male and female subjects. Based on the result of the current study, strategic supplementation with CFE might be of interest for recreational athletes competing in sports that have a large anaerobic component, such as sprinters and track cyclists. Although the reported effects are small, these observed differences may be of high relevance and impact, if these results of this study in recreational athletes can be extrapolated to highly trained sports professionals. In elite athletes, small differences may determine winning or losing a competition. Further research is needed to provide more insight into the effects of CFE supplementation in elite athletes, to substantiate CFE use as a nutritional ergogenic aid.

Although CFE supplementation increased anaerobic exercise performance in moderately trained subjects after supplementation with CFE, the underlying mechanism remains to be elucidated. A putative mechanism of action may be that CFE increases oxygen delivery to the muscles by upregulating $\mathrm{NO}$ with a subsequent vasodilation and increased blood flow response, thereby also increasing the removal of waste products, such as lactate. For instance, hesperetin, a metabolite from the citrus flavonoid hesperidin, has been associated with an increased NO release from endothelial cells and hesperidin supplementation in human subjects has been associated with improved endothelial function [12, 15, 27]. Furthermore, previous studies have demonstrated that hesperidin reduces oxidative stress levels by scavenging ROS and improving antioxidative capacity, which is especially beneficial for high intensity anaerobic exercise as anaerobic functioning leads to increased production of muscle fatigue associated end-products such as lactate and high ROS levels [28-30]. Another potential mechanism of action might involve modulation of mitochondrial metabolism. It has been demonstrated that treatment with hesperetin increases intracellular ATP by $33 \%$ and mitochondrial spare capacity (i.e. the difference between maximum and basal respiratory capacity) by $25 \%$ in myotubes [31], which could result in reduced oxidative stress levels and more ATP availability during high intensity exercise.

Some potential limitation of the current study design should be mentioned. Before the start of the study, a familiarization test was conducted to minimize learning effects of the WAnT exercise protocol. Nevertheless, a learning effect on the WAnT outcome cannot entirely be excluded, although the placebo arm did not show such learning effects over the consecutive tests, and the randomized design of the study will have minimized potential confounding of a potential learning effect. Another limitation of this study is that participants were not randomized based on training level, mode of training, intensity of training or frequency of training. All participants were moderately trained, but some variation in the aforementioned factors may have been present, resulting in high standard deviations. However, we did not observe significant differences in training hours per week or any of the baseline performance outcomes between groups. Furthermore, this study did not investigate the mechanisms which underlie the observed differences in anaerobic exercise performance. Blood collection and muscle biopsies would have given more insight into the effect of hesperidin on oxidative stress levels, inflammation levels and mitochondrial function [31]. These tests were not included in the current study in order to limit the test burden for participants.

\section{Conclusions}

This study shows that daily intake of CFE, a natural flavonoid containing supplement, resulted in increased anaerobic capacity and peak power output during high intensity exercise in moderately trained individuals without affecting the maximum heart rate. Future research needs to be performed to identify the underlying mechanisms that are affected by CFE supplementation.

\section{Supplementary Information}

The online version contains supplementary material available at https://doi. org/10.1186/s12970-020-00399-w.

Additional file 1: Table S1. Dietary intake at baseline, after 4 weeks and after 8 weeks of supplementation, with corresponding $p$-values.

\section{Abbreviations \\ 5sPP: 5 s Peak power; AP: Average power; ATP: Adenosine triphosphate; BMI: Body mass-index; Bpm: Beats per minute; CFE: Citrus flavonoid extract; HR: Heart rate; NO: Nitric oxide; ROS: Reactive oxygen species; SD: Standard deviation; SOD: Superoxide dismutase; WAnT: Wingate anaerobic test; WPM: Wattbike performance monitor}

\section{Acknowledgements}

The authors would like to thank Kevin $\mathrm{H}$. Wolfs for his input during the conceptualization phase and Joris Kretzers for his input and help with the revisions.

\section{Authors' contributions}

Conceptualization: FJT, YRS. Formal analysis: LEVI, YRS: Original draft preparation: LEVI. Critical revision: FJT, YRS, JMC. The author (s) read and approved the final manuscript.

\section{Funding}

This research was funded by BioActor BV.

Availability of data and materials

The datasets used and/or analyzed during the current study are available from the corresponding author on reasonable request. 


\section{Ethics approval and consent to participate}

This study was approved by the Medical Ethics Committee of Wageningen University, Wageningen, The Netherlands.

\section{Consent for publication}

Not applicable.

\section{Competing interests}

LEVI is a former employee of BioActor BV. YRS is a current employee of BioActor BV. The funders had no role in the design of the manuscript, writing of the manuscript, or in the decision to publish the results.

\section{Author details}

'BioActor BV, Gaetano Martinolaan 85, 6229 GS Maastricht, The Netherlands. ${ }^{2}$ Division Gastroenterology-Hepatology, Department of Internal Medicine, School of Nutrition and Translational Research in Metabolism (NUTRIM), Maastricht University, P.O. Box 616, 6200 MD Maastricht, The Netherlands. ${ }^{3}$ Department of Food Innovation and Health, Centre for Healthy Eating and Food Innovation, Maastricht University, 5911 BV Venlo, The Netherlands.

Received: 16 March 2020 Accepted: 7 December 2020

Published online: 06 January 2021

\section{References}

1. Knapik JJ, Steelman RA, Hoedebecke SS, Austin KG, Farina EK, Lieberman HR. Prevalence of dietary supplement use by athletes: systematic review and meta-analysis. Sports Med. 2016;46:103-23.

2. Cases J, Romain C, Marin-Pagan C, Chung LH, Rubio-Perez JM, Laurent C, et al. Supplementation with a polyphenol-rich extract, PerfLoad((R)), improves physical performance during high-intensity exercise: a randomized, double blind. Nutrients. 2017;9(4):421. https://doi.org/10.3390/ nu9040421.

3. Campbell B, Kreider RB, Ziegenfuss T, La Bounty P, Roberts M, Burke D, et al. International Society of Sports Nutrition position stand: protein and exercise. J Int Soc Sports Nutr. 2007:4:-8.

4. Hargreaves M, Hawley JA, Jeukendrup A. Pre-exercise carbohydrate and fat ingestion: effects on metabolism and performance. J Sports Sci. 2004:22:31-8.

5. Baker JS, McCormick MC, Robergs RA. Interaction among skeletal muscle metabolic energy systems during intense exercise. J Nutr Metab. 2010;2010: 905612

6. Gastin PB. Energy system interaction and relative contribution during maximal exercise. Sports Med. 2001:31:725-741.

7. Gomez-Cabrera MC, Domenech E, Vina J. Moderate exercise is an antioxidant: upregulation of antioxidant genes by training. Free Radic Biol Med. 2008;44:126-31

8. Simioni C, Zauli G, Martelli AM, Vitale M, Sacchetti G, Gonelli A, et al. Oxidative stress: role of physical exercise and antioxidant nutraceuticals in adulthood and aging. Oncotarget. 2018;9:17181-98.

9. Rothschild JA, Bishop DJ. Effects of dietary supplements on adaptations to endurance training. Sports Med. 2020;50:25-53.

10. Overdevest E, Wouters JA, Wolfs KHM, van Leeuwen JJM, Possemiers S. Citrus flavonoid supplementation improves exercise performance in trained athletes. J Sports Sci Med. 2018:17:24-30.

11. Bowtell J, Kelly V. Fruit-derived polyphenol supplementation for athlete recovery and performance. Sports Med. 2019;49:3-23.

12. Rizza S, Muniyappa R, lantorno M. Kim J-a, Chen H, Pullikotil P, et al. Citrus polyphenol hesperidin stimulates production of nitric oxide in endothelial cells while improving endothelial function and reducing inflammatory markers in patients with metabolic syndrome. The J Clin Endocrinol Metab. 2011;96:E782-E92

13. Bescos R, Sureda A, Tur JA, Pons A. The effect of nitric-oxide-related supplements on human performance. Sports Med. 2012;42:99-117.

14. Martinez-Noguera FJ, Marin-Pagan C, Carlos-Vivas J, Rubio-Arias JA, Alcaraz PE. Acute effects of hesperidin in oxidant/antioxidant state markers and performance in amateur cyclists. Nutrients. 2019;11.

15. Salden BN, Troost FJ, de Groot E, Stevens YR, Garces-Rimon M, Possemiers S, et al. Randomized clinical trial on the efficacy of hesperidin $2 S$ on validated cardiovascular biomarkers in healthy overweight individuals. Am J Clin Nutr. 2016;104:1523-33.
16. Zupan MF, Arata AW, Dawson LH, Wile AL, Payn TL, Hannon ME. Wingate anaerobic test peak power and anaerobic capacity classifications for men and women intercollegiate athletes. J Strength Cond Res. 2009;23:2598-604.

17. Evans JA, Quinney HA. Determination of resistance settings for anaerobic power testing. Can J Appl Sport Sci. 1981;6:53-6.

18. Inbar O, Bar-Or O, Skinners IS. The Wingate anaerobic test. Champaign, IL: Human Kinetics; 1996

19. Gattuso G, Barreca D, Gargiulli C, Leuzzi U, Caristi C. Flavonoid composition of Citrus juices. Molecules. 2007;12:1641-73.

20. Stevens $Y$, Winkens $B$, Jonkers $D$, Masclee $A$. The effect of olive leaf extract on cardiovascular health markers: a randomized placebo-controlled clinical trial. Eur J Nutr. 2020.

21. Davis JM, Carlstedt CJ, Chen S, Carmichael MD, Murphy EA. The dietary flavonoid quercetin increases VO(2max) and endurance capacity. Int J Sport Nutr Exerc Metab. 2010;20:56-62.

22. Deley G, Guillemet D, Allaert FA, Babault N. An acute dose of specific grape and apple polyphenols improves endurance performance: a randomized, crossover, double-blind versus placebo controlled study. Nutrients. 2017;9.

23. Oh JK, Shin YO, Yoon JH, Kim SH, Shin HC, Hwang HJ. Effect of supplementation with Ecklonia cava polyphenol on endurance performance of college students. Int J Sport Nutr Exerc Metab. 2010;20:72-9.

24. Richards JC, Lonac MC, Johnson TK, Schweder MM, Bell C. Epigallocatechin3-gallate increases maximal oxygen uptake in adult humans. Med Sci Sports Exerc. 2010:42:739-44

25. Strait JB, Lakatta EG. Aging-associated cardiovascular changes and their relationship to heart failure. Heart Fail Clin. 2012:8:143-64.

26. Bentley DJ, Ackerman J, Clifford T, Slattery KS. Acute and chronic effects of antioxidant supplementation on exercise performance. In: Lamprecht M, editor. Antioxidants in sport nutrition. Boca Raton, FL: CRC Press/Taylor \& Francis; 2015

27. Liu L, Xu DM, Cheng YY. Distinct effects of naringenin and hesperetin on nitric oxide production from endothelial cells. J Agric Food Chem. 2008:56:824-9.

28. Wilmsen PK, Spada DS, Salvador M. Antioxidant activity of the flavonoid hesperidin in chemical and biological systems. J Agric Food Chem. 2005:53:4757-61.

29. Estruel-Amades S, Massot-Cladera M, Garcia-Cerda P, Perez-Cano FJ, Franch $A$, Castell $M$, et al. Protective effect of hesperidin on the oxidative stress induced by an exhausting exercise in intensively trained rats. Nutrients. 2019;11.

30. de Oliveira DM, Dourado GK, Cesar TB. Hesperidin associated with continuous and interval swimming improved biochemical and oxidative biomarkers in rats. J Int Soc Sports Nutri. 2013;10:27.

31. Biesemann N, Ried JS, Ding-Pfennigdorff D, Dietrich A, Rudolph C, Hahn S, et al. High throughput screening of mitochondrial bioenergetics in human differentiated myotubes identifies novel enhancers of muscle performance in aged mice. Sci Rep. 2018:8:9408.

\section{Publisher's Note}

Springer Nature remains neutral with regard to jurisdictional claims in published maps and institutional affiliations.

Ready to submit your research? Choose BMC and benefit from:

- fast, convenient online submission

- thorough peer review by experienced researchers in your field

- rapid publication on acceptance

- support for research data, including large and complex data types

- gold Open Access which fosters wider collaboration and increased citations

- maximum visibility for your research: over $100 \mathrm{M}$ website views per year

At BMC, research is always in progress.

Learn more biomedcentral.com/submissions 